ARTICLE

https://doi.org/10.1038/s41467-019-12556-0

\title{
Coulomb-driven single defect engineering for scalable qubits and spin sensors in diamond
}

Tobias Lühmann ${ }^{1}$, Roger John¹, Ralf Wunderlich¹, Jan Meijer ${ }^{1} \&$ Sébastien Pezzagna*

Qubits based on colour centres in diamond became a prominent system for solid-state quantum information processing and sensing. But the deterministic creation of qubits and the control of their environment are still critical issues, preventing the development of a roomtemperature quantum computer. We report on the high creation yield of NV centres of $75 \%$ (a tenfold enhancement) by charge-assisted defect engineering, together with an improvement of their spin coherence. The method strongly favours the formation and negative charge state of the NV centres with respect to intrinsic diamond, while it hinders the formation of competing and perturbing defects such as di-vacancies or NVH complexes. We evidence spectrally the charge state tuning of the implantation-induced vacancies from $\mathrm{V}^{0}$ to $\mathrm{V}^{-}$, key element of this Coulomb-driven engineering. The generality of the method is demonstrated using several donors (phosphorous, oxygen and sulphur) and applying it to other centres ( $\mathrm{SnV}$ and $\mathrm{MgV}$ ) in diamond.

\footnotetext{
${ }^{1}$ Applied Quantum Systems, Felix-Bloch Institute for Solid-State Physics, University Leipzig, Linnéstraße 5, 04103 Leipzig, Germany.

*email: sebastien.pezzagna@uni-leipzig.de
} 
Q ubits in diamond based on the nitrogen-vacancy (NV) centre are widely implemented for a large range of applications like quantum information processing ${ }^{1}$, quantum sensing of magnetic fields ${ }^{2}$, electric fields ${ }^{3}$, or temperature $^{4}$ but also for nuclear magnetic resonance ${ }^{5}$ or even to answer fundamental questions in physics ${ }^{6,7}$. The fabrication and reproducibility of scalable devices requires (and still lacks) sample homogeneity and creation yields of the qubits close to $100 \%{ }^{8}$, but as well, the control and engineering of their environment ${ }^{9}$. The addressing using ion implantation provides high spatial resolution $^{10}$, placement accuracy ${ }^{11}$, and even deterministic countable single ions ${ }^{12-15}$. However, the formation of impurity-vacancy centres $(\mathrm{NV}, \mathrm{SiV}, \mathrm{SnV}, \ldots)$ using a thermal treatment ${ }^{16,17}$ enabling the mobility of the implantation-induced vacancies ${ }^{18,19}$ is a statistical process of generally low efficiency (a few \% or less $^{20}$ ). It is known that a large part of these vacancies are lost by recombination with carbon interstitials, by formation of divacancies or other complexes (vacancy chains, $\mathrm{VH}$ ) or by diffusion to dislocations or to the diamond surface for the shallow ones. It was moreover shown that the proportion of vacancy complexes (with respect to isolated vacancies) increases with the atomic mass of the implanted ions ${ }^{9}$ and that suitable thermal treatments enable the dissociation of vacancy chains ${ }^{21}$. Efforts were also made to increase the $\mathrm{NV}$ yield (for example by co-implantation of carbon ${ }^{22}$, post-irradiation with electrons or protons to produce additional vacancies ${ }^{23,24}$ or more recently very efficiently by laser writing ${ }^{25}$ ) and to understand the NV formation process 26,27 . Not only for scalability purposes, high creation yields would ensure a clean environment free of unconverted and perturbing atoms, for example, nitrogen in the case of $\mathrm{NV}$ centres $^{28}$, whereas a close-by donor (such as nitrogen or phosphorous) is necessary to keep the charge state stability ${ }^{29-32}$ necessary for most applications.

Furthermore, hydrogen is by orders of magnitude the most abundant impurity in ultrapure IIa CVD diamonds (owing to the hydrogen plasma assisted growth) but its exact concentration and role in colour centre formation are difficult to evaluate. Though, it was shown that the $\mathrm{NVH}$ concentration in CVD grown layers is typically at least one order of magnitude larger than the NV centres concentration, whatever the nitrogen content ${ }^{33}$, and that the formation energy of the NVH defect is $\sim 1 \mathrm{eV}$ lower than that of the NV centre ${ }^{28}$. The low yields of implanted NV centres may also be owing to hydrogen diffusion and NV passivation $9,34,35$ during the thermal treatments. Note that besides temperature ${ }^{34}$, hydrogen (deuterium) diffusion is known to also strongly depend on defect concentration, doping 36,37 , or electric bias ${ }^{38}$.

Recently, using a sacrificial B-doped diamond layer led to a better formation yield of shallow NV centres (from 1 to $2 \%)^{39}$. This was attributed to the charging of single vacancies (otherwise mostly neutral in intrinsic diamond), which was expected to hinder the formation of di-vacancies. Also, the assumption that the chemical potential is pinned down enough to charge vacancies positively would then imply that substitutional nitrogen atoms are also positively charged, which may eventually hinder the formation of the NV centres (if one agrees with the previous electric repulsion argument leading to less di-vacancies). Ab initio calculations of the adiabatic charge transition levels of $\mathrm{V}$ and $\mathrm{N}$ can be found in ${ }^{27}$.

On the other hand, we intend to charge the vacancies negatively $\mathrm{V}^{-}$, whereas having positively charged nitrogen $\mathrm{N}^{+}$, which may enhance the NV centre formation even more (still preventing $\mathrm{V}-\mathrm{V}$ formation) using Coulomb forces. This is one of the main questions addressed in this work. Such a situation is likely accessible using a suitable n-type doping and other questions we address are whether using phosphorous implantation can provide enough electrically active donors (phosphorous-doped CVD grown (100) diamond is generally significantly compensated 40,41 owing to the presence of acceptor defects), how alternative donors compare with phosphorous ${ }^{42,43}$, and how the qubits coherence time compares with intrinsic diamond.

\section{Results}

Evidence of vacancy charging by efficient phosphorous doping. We study the creation efficiency, thermal stability, and charge states of implanted $\mathrm{NV}, \mathrm{SnV}$, and Mg-related centres as a function of the doping level of diamond, using the most commonly employed acceptor (boron, $E_{\mathrm{a}}=0.37 \mathrm{eV}$ ) and donor (phosphorous, $E_{\mathrm{d}}=0.57 \mathrm{eV}$ ) and in a second step alternative donors (oxygen, $E_{\mathrm{d}}=0.32 \mathrm{eV}^{42}$ and Sulphur, $E_{\mathrm{d}}=0.77 \mathrm{eV}^{43}$ ). We firstly give evidence of the control of the charge state of single vacancies after implantation (and before thermal annealing), which we then systematically correlate to the formation efficiency of the different colour centres. The methodology consists in a sequence of ion implantations (doping and colour centres), thermal annealings and optical and spin characterisations using fluorescence confocal microscopy, as resumed in Fig. 1. The preliminary experiment consists in the implantation of carbon ions, repeated into the different doped areas in order to produce vacancies, measure their charge state (before thermal annealing) and evaluate the efficiency of the phosphorous and boron doping. For this, we follow (as in Fig. 2b) the characteristic optical fluorescence of isolated neutral vacancies $\mathrm{V}^{0}$ with zero-phonon-line (ZPL) at $741 \mathrm{~nm}^{17}$. The main result here is that Phosphorous doping induces a negative charging of the vacancies into $\mathrm{V}^{-}$(the absorption band of negatively charged vacancies $\mathrm{V}^{-}$with ZPL at $394 \mathrm{~nm}$ (ND1) ${ }^{17}$ cannot be observed here and does not contribute any fluorescence). Note that, as shown by Collins ${ }^{44}$, the charge state of a defect centre in diamond is depending on the presence of a closeby donor ${ }^{31}$ or acceptor rather than the position of the chemical potential, which is moreover difficult to calculate in an insulating material under light illumination. Here, the absence of $\mathrm{V}^{0}$ fluorescence means therefore that there are enough electrically active phosphorous atoms to provide electrons to the single vacancies, or in a semiconductor view, to pin up the chemical potential above the $\mathrm{V}(0 /-)$ charge transition of the single vacancies. As seen in Fig. 2, the $\mathrm{V}^{0}$ fluorescence from the $\mathrm{C}$ implantation is strengthened in the B-doped area whereas it is suppressed in the $\mathrm{P}$-area (fully tuned to $\mathrm{V}^{-}$) with respect to intrinsic diamond. For the highest fluence of $2 \times 10^{13} \mathrm{~cm}^{-2}$ (close to the amorphisation threshold), the implantation damage results in a lower fluorescence than the background of the unimplanted diamond. In the B-doped region however, this higher damage already occurs for the $2 \times 10^{12} \mathrm{~cm}^{-2}$ fluence. These damaged areas reveal the formation of vacancy complexes or even voids, also predicted by molecular dynamics simulations ${ }^{9}$. Their occurrence at lower fluences in the B-doped region is owing to the fact that in this region, where the $\mathrm{V}^{0}$ charge state is strengthened, the isolated vacancies can group together more easily. By opposition, in the P-doped area where the vacancies are negatively charged, the high damage is only visible at a $2 \times 10^{13} \mathrm{~cm}^{-2} \mathrm{flu}-$ ence, revealing that vacancy clustering is reduced. The intrinsic region presents an intermediate situation for which both $\mathrm{V}^{0}$ and $\mathrm{V}^{-}$are present, indicating that the chemical potential lies close to the $\mathrm{V}(0 /-)$ transition level during the measurement (under laser excitation). Note that no fluorescence is known from the positively charged vacancy $\mathrm{V}^{+}$. These results confirm that isolated vacancies are preserved when they are charged, whereas vacancy complexes can form more easily when they are neutral. The consequences of this on the impurity-vacancy defects formation will be discussed in the next sections. 
$\mathbf{a}$
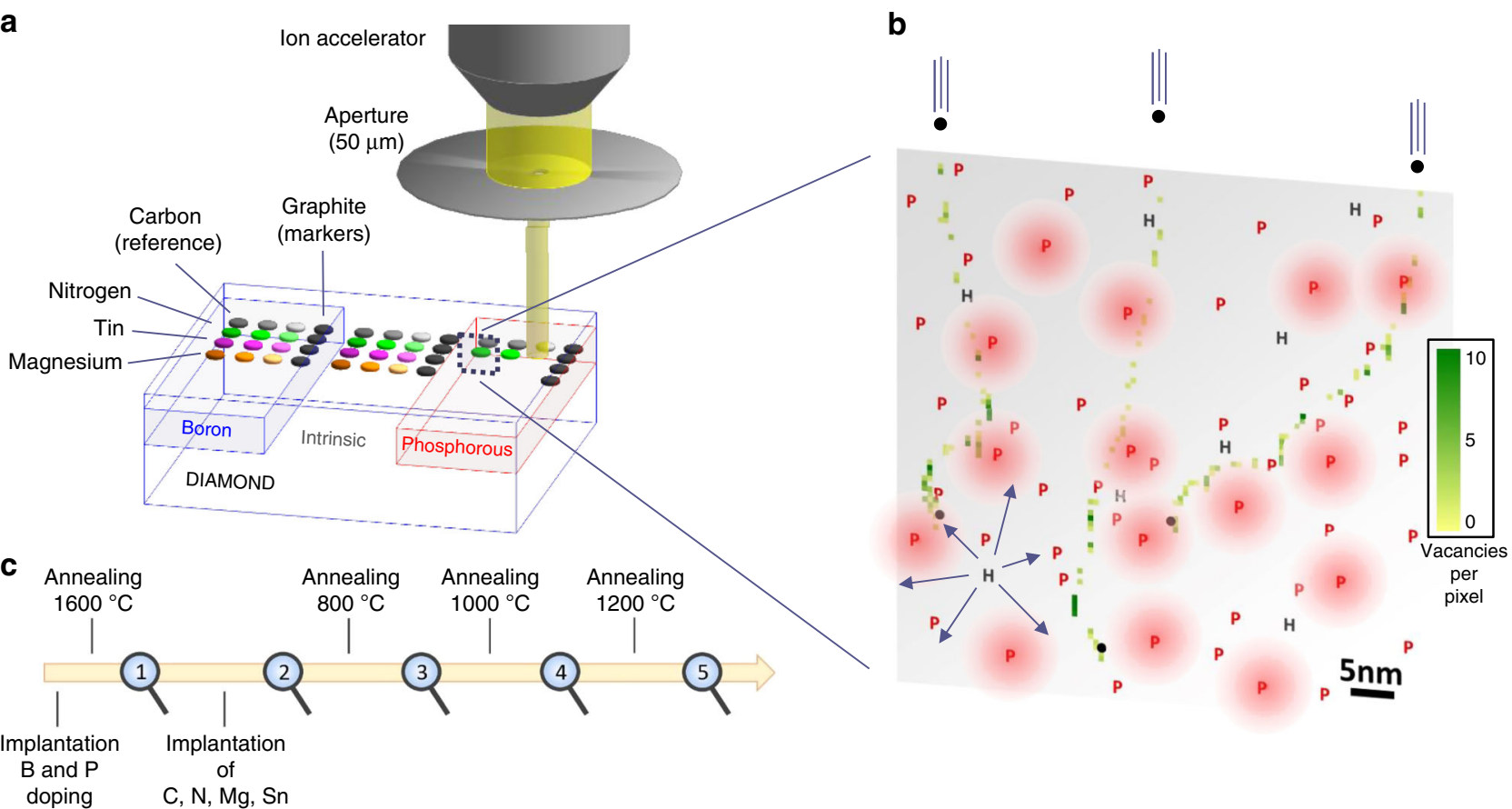

Fig. 1 Colour centre implantation within three doped regions. a Scheme of the diamond sample (quantum grade from Element 6) prepared with intrinsic, ptype and $n$-type areas (using boron and phosphorous implantation with $[\mathrm{P}]$ and $[\mathrm{B}] \sim 3 \times 10^{18} \mathrm{~cm}^{-3}$, see supplementary information) and implanted with a series of different chemical elements: carbon (as reference) and nitrogen, tin, and magnesium to produce, NV, SnV and MgV centres. The depth of the centres $(\sim 50 \mathrm{~nm})$ is controlled by the implantation energy and fits with the dopant depth profiles (see supplementary information). b Illustration of the sample status after implantation in the Phosphorous-doped region, as represented by a $60 \times 60 \mathrm{~nm}^{2}$ cross-section. The implantation-induced vacancies are simulated by SRIM. The density is given in number of vacancies per pixel of size $0.6 \times 0.6 \mathrm{~nm}^{2}$. The phosphorous atoms are represented by the red $P$ letters, with inter-distance corresponding to $[P] \approx 3 \times 10^{18} \mathrm{~cm}^{-3}$. The red halos represent substitutional $\mathrm{P}$ active donors which are non-passivated and nonself-compensated. Hydrogen atoms are also represented at a density of $1 \times 10^{17} \mathrm{~cm}^{-3}$, with arrows accounting for their large diffusion $\left(\sim 6 \times 10^{7} \mathrm{~nm} \mathrm{~s}^{-1}\right.$ at $800^{\circ} \mathrm{C}$ in lla diamond ${ }^{34}$ ). Less than 2.5 nitrogen and 0.5 boron atoms are expected within this section for native concentration $[\mathrm{N}]<5 \mathrm{ppb}$ and $[\mathrm{B}]<1 \mathrm{ppb}$ $\mathrm{NVH}, \mathrm{V}_{2}, \mathrm{~V}_{\mathrm{x}}, \mathrm{VH}$, and $\mathrm{PV}$ are the main competing defects to NV formation, whereas $\mathrm{PH}$ may passivate the donors. NVN forms at much higher temperatures and/or [N] densities owing to the low diffusion of nitrogen $\left(\sim 1.7 \times 10^{-3} \mathrm{~nm}^{2} . \mathrm{s}^{-1}\right.$ at $1600^{\circ} \mathrm{C}$ in ref. $\left.{ }^{9}\right)$. c Time sequence of the different steps of the study. The magnifiers symbolise imaging and spectroscopy

Note that an estimation of the efficiency of implanted phosphorous as an active donor (in substitutional site, nonpassivated, and non-compensated) by comparing the number of created vacancies using SRIM simulations ${ }^{45}$ and the number of phosphorous atoms previously implanted indicates that the phosphorous atoms are efficiently placed in substitutional sites and that the formation of complexes such as $\mathrm{PH}$ or $\mathrm{PV}$ (passivating or compensating the donor, respectively) ${ }^{46,47}$ is likely low. An occupation of substitutional sites of $70 \%$ by implanted phosphorous after thermal annealing was measured using internal channelling measurements ${ }^{48}$. Recently, single optically active defects likely related to phosphorous were reported in diamond with a creation yield of $10^{-4}$ to $10^{-59}$ but of still unknown structure.

NV centres: doping-dependent creation efficiency. Before annealing, the fluorescence of vacancies induced by nitrogen implantation (Fig. 3b) reveals, as for carbon atoms, that the vacancies are fully tuned to $\mathrm{V}^{-}$when the phosphorous doping is increased, whereas $\mathrm{V}^{0}$ fluorescence is clearly visible in the intrinsic and the boron-doped areas. The pre-annealing situation is illustrated in Fig. 1b, with average distances between impurities given at scale (and extrapolated for a 2D cross-section) for a nitrogen fluence of $1 \times 10^{11} \mathrm{~cm}^{-2}$ and concentrations $[\mathrm{P}] \approx$ $12 \mathrm{ppm},[\mathrm{H}] \approx 600 \mathrm{ppb},[\mathrm{N}]<5 \mathrm{ppb}$ and $[\mathrm{B}]<1 \mathrm{ppb}$. Note that the donor effect of nitrogen itself can be neglected here because $\sim 160$ vacancies are created for each implanted nitrogen.
Before the $800^{\circ} \mathrm{C}$ annealing step is conducted, the vacancies thus have different charge states depending on the doped area. The nitrogen atoms, when placed in substitutional sites, are expected to be in $\mathrm{N}^{+}$state (vacancies are compensating acceptors for nitrogen and in large majority before annealing, Fig. $2 \mathrm{c}$ (see supplementary Figure 10)). The consequence of this can now be evaluated from the NV centres fluorescence in Fig. $3 \mathrm{a}$ after $800^{\circ} \mathrm{C}$ annealing. The NV creation yield is calculated for each implantation spot and plotted in Fig. 3f. For intrinsic diamond, it is in the range 6-8\%, which is typical for this implantation energy $y^{9,20,22}$ (and the same for either as-grown or polished diamond). Interestingly, the B- and P-doping give rise to very different behaviours: the creation yield is reduced for B-doping (about twice lower), whereas it is enhanced for Pdoping. The highest effect of phosphorous is observed in the low nitrogen fluence regime, for which the ratio "dopants over other defects" is maximum. The NV yield has a threefold increase up to $22.4 \%$ under the effect of phosphorous, as also highlighted by Fig. 3d, e. This corresponds to the ideal situation during thermal annealing for which the involved impurities are mostly in the following charge states: $\mathrm{V}^{-}, \mathrm{N}^{+}, \mathrm{P}^{+}$, and $\mathrm{H}^{-}$(hydrogen in the bond-centred site has acceptor behaviour with $\mathrm{H}(0 /-) \sim 2 \mathrm{eV}$ below the conduction band ${ }^{49}$ ), thus likely hindering the formation of competing $\mathrm{NVH}, \mathrm{V}_{2}$, and $\mathrm{VH}$ defects, in favour of $\mathrm{NV}$. Note that the slightly enhanced $\mathrm{NV}$ yield observed in reference ${ }^{39}$ using boron doping is owing to the high doping level $\left(\left[\mathrm{N}_{\mathrm{A}}\right]=10^{20} \mathrm{~cm}^{-3}\right)$, which likely induces a $\mathrm{V}^{+}$charge state precluding the formation of di-vacancies, whereas the implanted boron concentration used here 


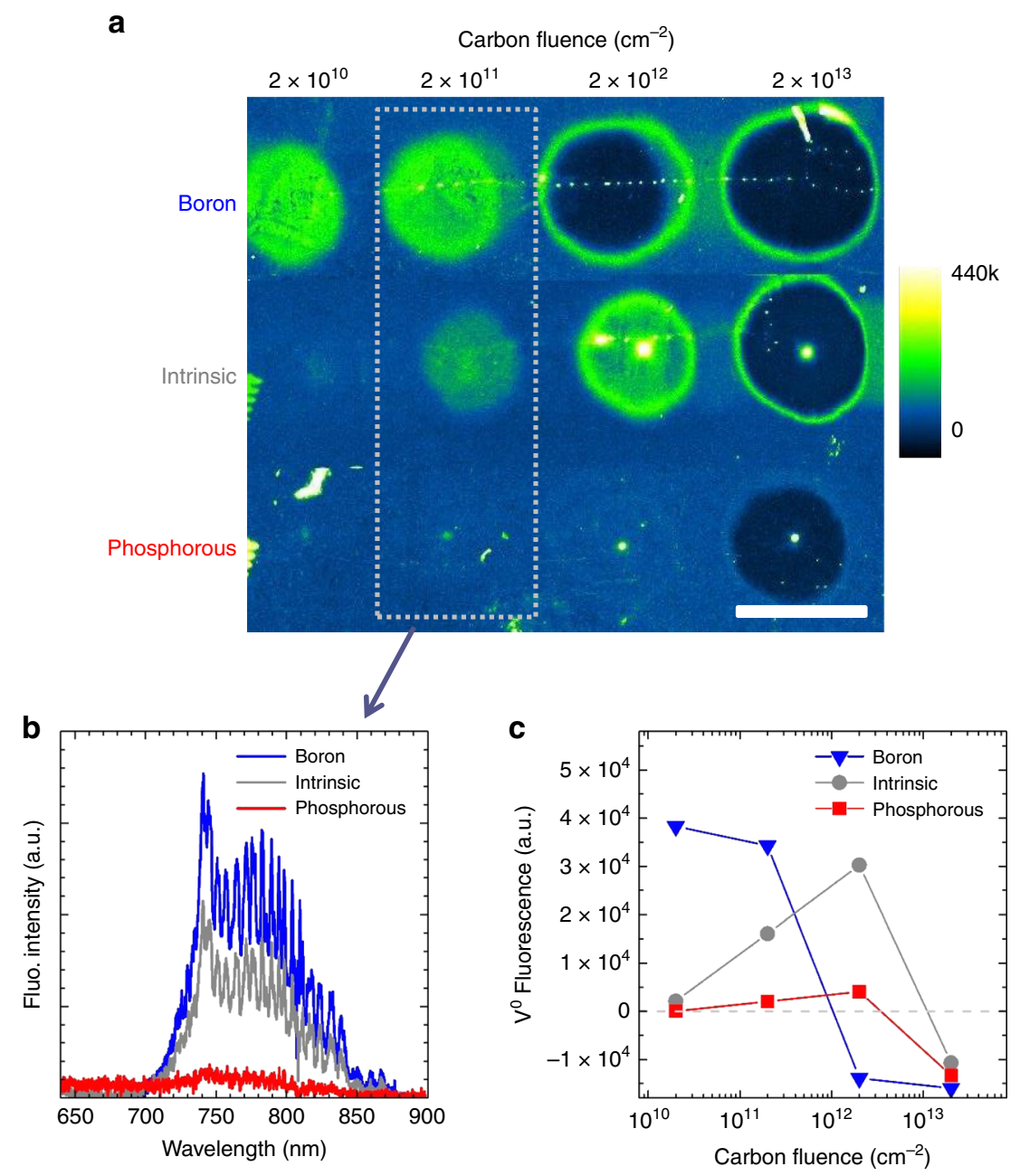

Fig. 2 Charge state tuning of single vacancies by diamond doping. a Confocal fluorescence scans of the carbon implantations with fluences in the range $2 \times$ $10^{10}-2 \times 10^{13} \mathrm{~cm}^{-2}$, repeated within the three different doped regions: $p$-type, intrinsic and $\mathrm{n}$-type with $[\mathrm{B}]$ and $[\mathrm{P}] \sim 3 \times 10^{18} \mathrm{~cm}-3$. The excitation wavelength is $532 \mathrm{~nm}$ and the detection is above $650 \mathrm{~nm}$ to record the signal from neutral vacancies $V^{0}$ (GR1 centre, ZPL at $741 \mathrm{~nm}$ ). The scale bar is $50 \mu \mathrm{m}$. The bright dots visible in some implanted spots are owing to a focused electron beam during cathodoluminescence measurements (see supplementary Figure 11). b Fluorescence spectra recorded in the three doped areas for the $2 \times 10^{11} \mathrm{~cm}^{-2}$ carbon fluence (acquisition by scanning a $10 \times$ $10 \mu \mathrm{m}^{2}$ area at the centre of each spot). The characteristic emission from the GR1 is recognised. The absence of GR1 fluorescence in the P-doped area is owing to charging up of the vacancies in their negative state $V^{-}$. The charge state transition levels of vacancies can be found in ${ }^{27} \mathbf{c} A v e r a g e V^{0}$ fluorescence intensity (with respect to the background fluorescence level given as the zero level) as a function of the carbon fluence and diamond doping

$\left(2 \times 10^{18} \mathrm{~cm}^{-3}\right)$ stabilises $\mathrm{V}^{0}$, thus leading to more di-vacancies and a lower NV yield.

Tin-vacancy and Mg-related centres. This Coulomb-driven defect engineering has also been applied to $\mathrm{SnV}$ and $\mathrm{Mg}$ related $^{9}$ centres to establish whether the previous results are specific to NV centres or possibly a more general trend for impurity-vacancy centres. Recently, it was shown that high yields of $\mathrm{SiV}^{0}$ centres were obtained in boron-doped diamond ${ }^{50}$. Our method (Fig. 1a) allows the direct comparison of different impurity-vacancy defects in exactly the same conditions: same diamond sample, same doping levels, same thermal annealing and same measurement parameters, at the exception of possible spatial inhomogeneity within the sample. The properties of column IV-vacancy ( $\mathrm{SiV}, \mathrm{GeV}, \mathrm{SnV}$, and $\mathrm{PbV}$ ) centres arouse growing interest ${ }^{51-54}$ and the creation of single $\mathrm{SnV}$ centres was reported recently, based on $\mathrm{Sn}$ implantation and thermal annealing ${ }^{51,52}$. Creation yields of implanted $\mathrm{SnV}$ centres were reported in the range $1-5 \%$ (in undoped diamond), comparable to what is obtained for $\mathrm{SiV}^{55}$ or $\mathrm{GeV}^{56}$. Owing to the high damage induced by the large $\mathrm{Sn}$ atom ${ }^{9}$, higher temperatures (also using high pressure to prevent graphitisation) were preferred, which indeed led to reduced ZPL width ${ }^{52}$ (indicative of a less defected environment) and to the removal of the fluorescence peaks at $595 \mathrm{~nm}$ and $646 \mathrm{~nm}$ observed for temperatures below $1500^{\circ} \mathrm{C}$. Figure $4 \mathrm{a}$ is a fluorescence plot of the $\mathrm{Sn}$ implantation spots, after the temperature step at $1200^{\circ} \mathrm{C}$. The corresponding spectra in Fig. 4c confirm the formation of $\mathrm{SnV}$, with the characteristic ZPL at $620.3 \mathrm{~nm}$. The main result is that the highest $\mathrm{SnV}$ fluorescence is found in the phosphorous region (Fig. 4b), with a creation yield of $8.6 \%$, which is more than three times larger than in the intrinsic (2.5\%) and the B-doped (1.6\%) areas. These results reveal the same behaviour as what was obtained for the NV centres, which can be explained by a more efficient formation of $\mathrm{SnV}$ together with a reduced formation of competing centres. Note that unlike the NV, the structure of the SnV centre has $\mathrm{D}_{3 \mathrm{~d}}$ symmetry (like the $\mathrm{SiV}$ and $\mathrm{GeV}$ ), with the $\mathrm{Sn}$ atom sitting between two vacancies in an interstitial position. A discussion about the possible charge states of the $\mathrm{SnV}$ centre is found in the supplementary Figure 9. 
a
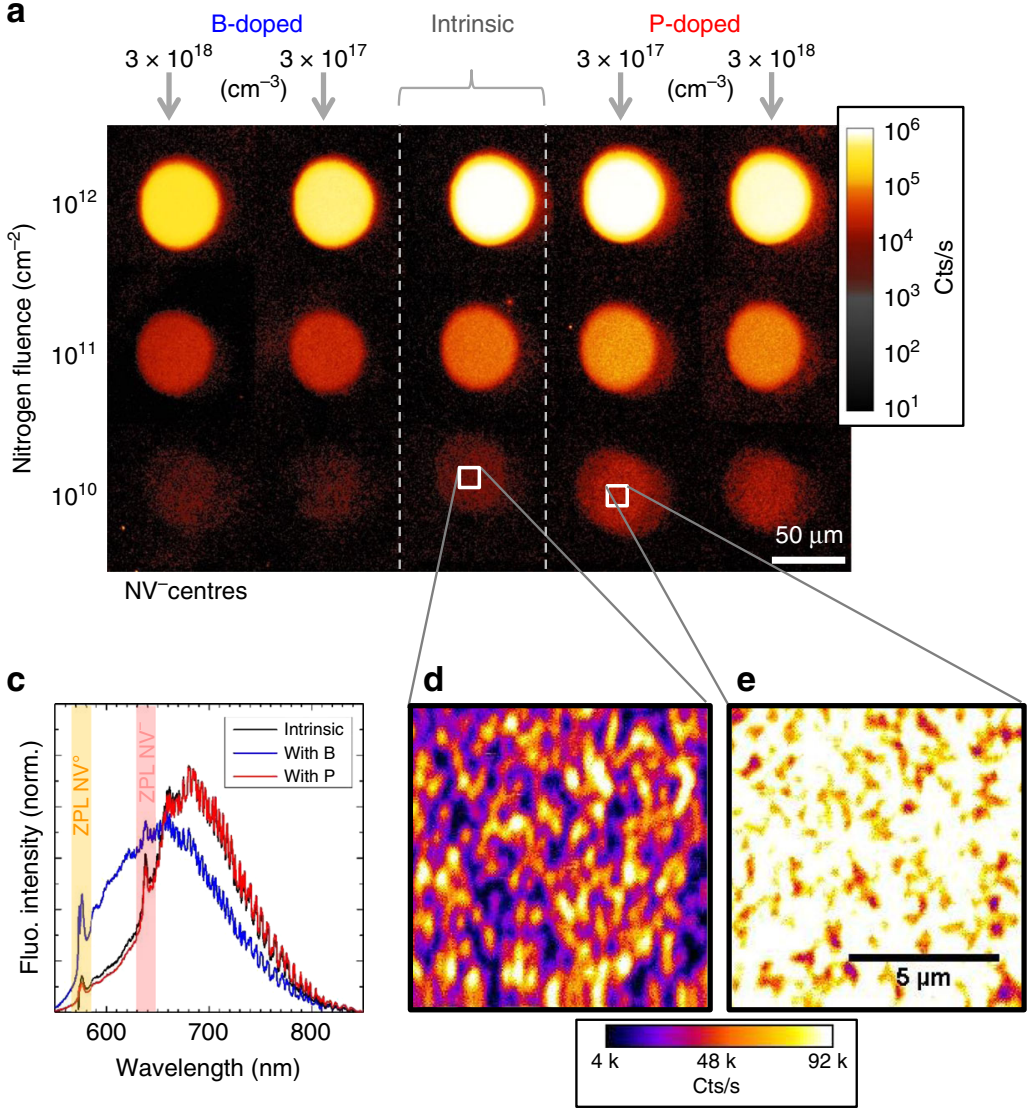

b
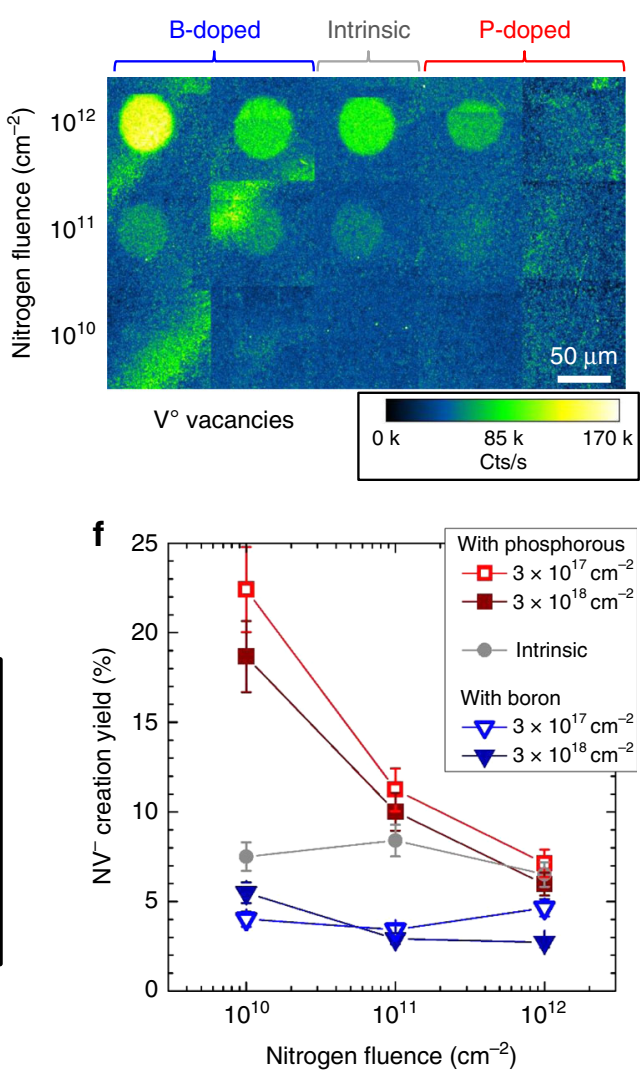

Fig. 3 Doping-dependent NV centre formation at $800^{\circ} \mathrm{C}$. a Confocal fluorescence scans of the nitrogen implantations in the different doping areas recorded after thermal annealing at $800^{\circ} \mathrm{C}$ for $4 \mathrm{~h}$. The laser excitation is $532 \mathrm{~nm}$ and a longpass filter $600 \mathrm{~nm}$ is used to record the NV centres fluorescence. The fluorescence intensity is in logarithmic scale. $\mathbf{b}$ Confocal fluorescence scans of the same areas measured directly after nitrogen implantation and before annealing. A longpass filter $700 \mathrm{~nm}$ is used to record $\mathrm{V}^{0}$ (GR1) fluorescence. c Normalised fluorescence spectra of the NV centres fluorescence ( $10^{12} \mathrm{~cm}^{-2}$ fluence) showing the effect of the boron and the phosphorous doping with respect to the intrinsic region, respectively. The ZPL of $\mathrm{NV}^{0}(575 \mathrm{~nm})$ and of $\mathrm{NV}^{-}(638 \mathrm{~nm})$ are highlighted. Contrarily to boron (giving more NV0), the effect of phosphorous on the NV charge state is minimal because for a $7.5 \%$ yield there are already 13 unconverted $\mathrm{N}$ donors for each NV centre. $\mathbf{d}$ and $\mathbf{e}$ are closer scans (with linear fluorescence scale) of two regions of interest of low nitrogen fluence $\left(10^{10} \mathrm{~cm}^{-2}\right)$ for the intrinsic and phosphorous-doped diamond $\left([\mathrm{P}] \sim 3 \times 10^{17} \mathrm{~cm}^{-3}\right)$. $\mathbf{f ~ N V ~ c e n t r e s ~ c r e a t i o n ~ y i e l d ~}$ vs nitrogen fluence and doping type/level, deduced from $\mathbf{a}, \mathbf{d}$, and $\mathbf{e}$. The Phosphorous doping induces up to a threefold yield improvement from $7.5 \%$ to $22.4 \%$. The error bars represent the measurement uncertainties derived from the yield formula given in supplementary Equation 3

Concerning the Mg-related centres, which are believed to involve vacancies (because single centres exhibit a strong polarisation anisotropy in excitation, see supplementary Figure 8), the fluorescence measured from the implantation spots in Fig. $4 \mathrm{~d}$ shows the characteristic emission at $557.4 \mathrm{~nm}^{9}$ (Fig. $4 \mathrm{f}$ ). The corresponding charge state is still unknown. No doping dependence is observed in the $\mathrm{MgV}$ spectra (between $550 \mathrm{~nm}$ and $900 \mathrm{~nm}$ and at all temperatures) at the exception of the weak feature at $544.5 \mathrm{~nm}$ in the P-doped region). Like in the case of the $\mathrm{NV}$ and $\mathrm{SnV}$ centres, the Mg-centre emission is at strongest in the P-doped region, here with up to a sixfold improved yield (for the $3 \times 10^{10} \mathrm{~cm}^{-2} \mathrm{Mg}$ fluence) from $7.8 \%$ in the intrinsic area to $48.4 \%$ in the P-doped area (as seen in Fig. 4e). This result further supports the assumption of a vacancy involved in the centre. Note that it cannot be excluded here that a less negative state (than the $557.4 \mathrm{~nm} \mathrm{ZPL}$ ) of the $\mathrm{MgV}$ centre may be optically inactive (or above $900 \mathrm{~nm}$ ) and lead to the higher yield of the $557.4 \mathrm{~nm} \mathrm{MgV}$. Further studies such as ab initio calculations or active electrical tuning ${ }^{57}$ of single centres are necessary.

Temperature evolution. The temperature dependence of the centres density (Fig. 5) confirms the higher yields in phosphorous- doped diamond in all cases. However, although the $\mathrm{SnV}$ and $\mathrm{MgV}$ population keeps increasing with the annealing temperature, the NV centres behave differently. The NV yield drops strongly (more than one order of magnitude) for intrinsic and B-doping between $800^{\circ} \mathrm{C}$ and $1000{ }^{\circ} \mathrm{C}$, whereas it still increases (from 11 to $14 \%$ ) in the P-doped area, before it finally decreases at $1200^{\circ} \mathrm{C}$. This striking difference cannot be explained by $\mathrm{N}$ diffusion and formation of NVN (H3 centres with ZPL at $503 \mathrm{~nm}$ ), which was not measured here and is only significant for much higher nitrogen densities and/or temperatures ${ }^{9}$. NV dissociation is expected to occur at higher temperatures as it was shown that a temperature of $1150^{\circ} \mathrm{C}$ (90 min) is not high (long) enough to induce a change of the $\mathrm{NV}$ direction (owing to vacancy diffusion) ${ }^{9}$. We attribute this precocious loss of NV centres in the intrinsic and boron-doped areas to their passivation by hydrogen ${ }^{9,34,35}$, knowing that the charge state and diffusion of hydrogen are doping-dependent ${ }^{36,37}$ and that the NV centres are preserved by the phosphorous doping.

These results indicate that, contrarily to $\mathrm{SnV}$ and $\mathrm{MgV}$ centres, a compromise is likely to be found for the optimal formation of $\mathrm{NV}$ centres upon thermal annealing, between forming NV centres, recovering a healed crystal structure without vacancy complexes ${ }^{21}$ and avoiding hydrogen passivation. The temperature 


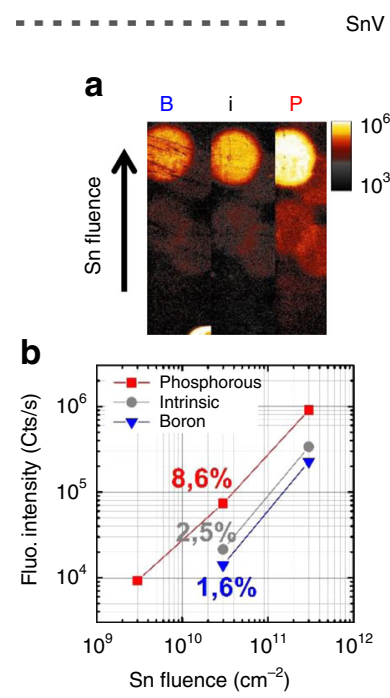

nV centres

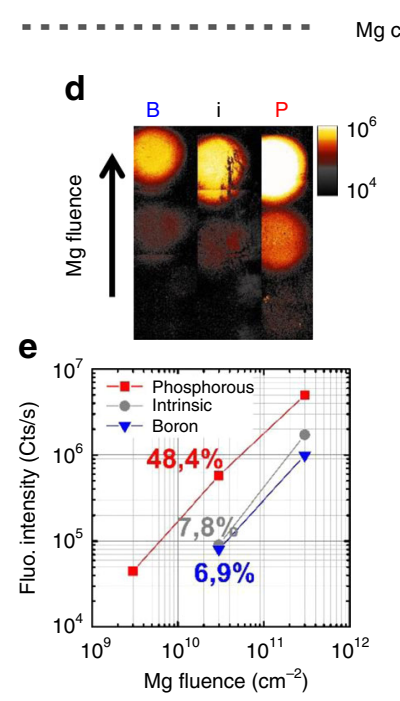

Mg centres

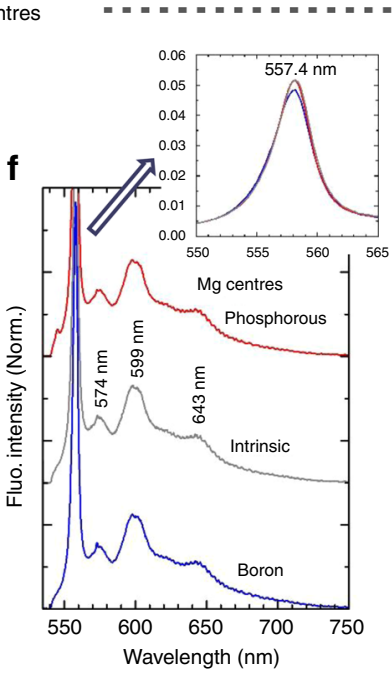

Fig. 4 Doping-dependent $\mathrm{SnV}$ and $\mathrm{MgV}$ centre formation at $1200^{\circ} \mathrm{C}$. a Confocal fluorescence scans of the SnV centres implanted in the different doping areas, recorded after the last thermal annealing at $1200^{\circ} \mathrm{C}$ for $4 \mathrm{~h}$. The fluorescence intensity is in logarithmic scale. The laser excitation is $532 \mathrm{~nm}$ and the detection with a longpass filter $550 \mathrm{~nm}$. b Plot of the fluorescence intensity vs fluence, for the different doping conditions. The corresponding creation yields are indicated. c Normalised fluorescence spectra measured from the different Sn-implanted areas. The spectra are recorded within the $10 \times 10 \mu m^{2}$ central region of each implanted spot. The spectra are background corrected (with references taken from $10 \times 10 \mu \mathrm{m}^{2}$ unimplanted neighbouring areas). $\mathbf{d}$, e, and $\mathbf{f}$, same for Mg-related centres

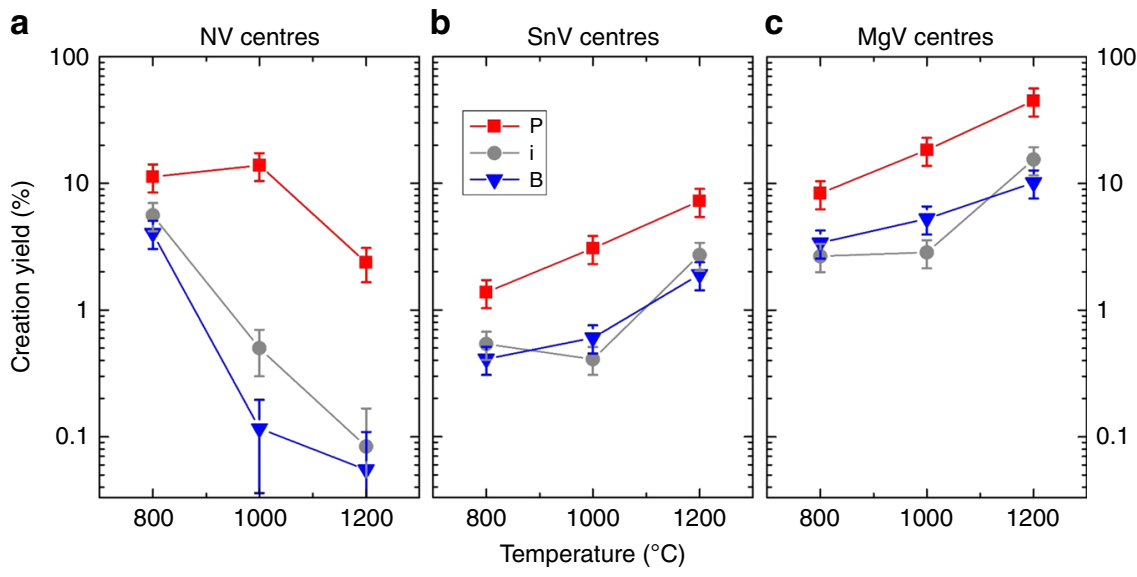

Fig. 5 Temperature evolution of colour centre population. a Creation yield (ratio of NV density and implanted nitrogen fluence, here $3 \times 10^{11} \mathrm{~cm}^{-2}$ ) of NV centres measured after the different consecutive annealing steps of $4 \mathrm{~h}$ each and for the different doping conditions. The effective yield enhancement between intrinsic and P-doped diamond is of three at $800^{\circ} \mathrm{C}$, whereas it is of $\sim 27$ at $1000^{\circ} \mathrm{C}$. b Same graph for $\mathrm{SnV}$ centres $\left(\mathrm{Sn}\right.$ fluence of $3 \times 10^{11} \mathrm{~cm}-2$ ). c Same graph for $\mathrm{MgV}$ centres $\left(\mathrm{Mg}\right.$ fluence of $3 \times 10^{11} \mathrm{~cm}^{-2}$ ). The error bars represent the measurement uncertainties derived from the yield formula given in supplementary Equation 3

range $900{ }^{\circ} \mathrm{C}-1100^{\circ} \mathrm{C}$ seems to be adequate for diamond predoped with donors, but the hydrogen native concentration and the annealing dynamics and times are also important parameters.

Oxygen and Sulphur as efficient donors. Another sample was prepared as in Fig. 1, but with the additional donors oxygen and sulphur, and annealed at $1200^{\circ} \mathrm{C}$ instead of $1600^{\circ} \mathrm{C}$. Nitrogen was then implanted and annealed at $800^{\circ} \mathrm{C}$ for $4 \mathrm{~h}$. The creation yield of NV centres (Fig. 6) increases of about one order of magnitude in the donor areas with respect to intrinsic diamond and reaches $69.3 \%$ for oxygen and $75.3 \%$ for Sulphur. The ensemble fluorescence spectra reveal that Sulphur induces also the most negative $\mathrm{NV}^{-}$average charge state. For phosphorous, the reduced annealing temperature of $1200^{\circ} \mathrm{C}$ used here gives a three times better yield (59.7\%) than for the previous $1600^{\circ} \mathrm{C}$ treatment. This can be due to an increased compensation and passivation of the donors at higher temperature, as observed for phosphorous-doped CVD grown layers when post-annealed at high temperature ${ }^{40}$.

Finally, the spin properties of the NV centres were studied in all areas and $\mathrm{T}_{2}$ and $\mathrm{T}_{2}{ }^{*}$ coherence times of several $\mathrm{NV}$ centres are plotted in Fig. $6 \mathrm{c}$, as a function of the concentration of implanted nitrogen and donors. The green lines represent the standard observed dependence of $\mathrm{T}_{2}$ and $\mathrm{T}_{2}{ }^{*}$ on the nitrogen concentration for as-grown $\mathrm{N}$-doped diamond ${ }^{28}$. In the intrinsic area, the coherence times are shorter, due to residual implantation defects, especially di-vacancies ${ }^{21}$. Among all donors, $\mathrm{T}_{2}$ times are at best in the sulphur area, indicating that the excess of (likely) neutral donors-about one NV centre for ten donors-leads to decoherence in the case of phosphorous but does not, or much less, in the case of sulphur. The coherence times are improved (reaching up to $\sim 100 \mu \mathrm{s}$ ) with respect to an equivalent nitrogen concentration of about 7 ppm (typically $20 \mu \mathrm{s}$ ). 
a

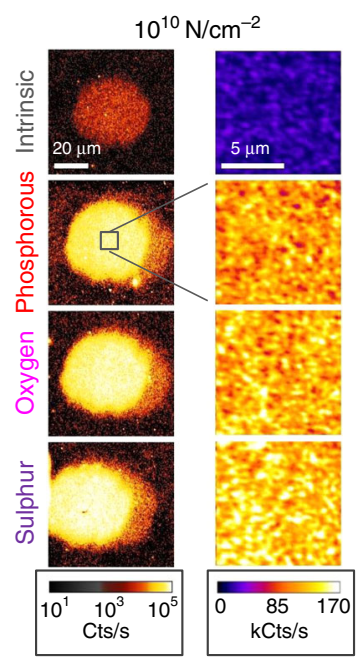

b
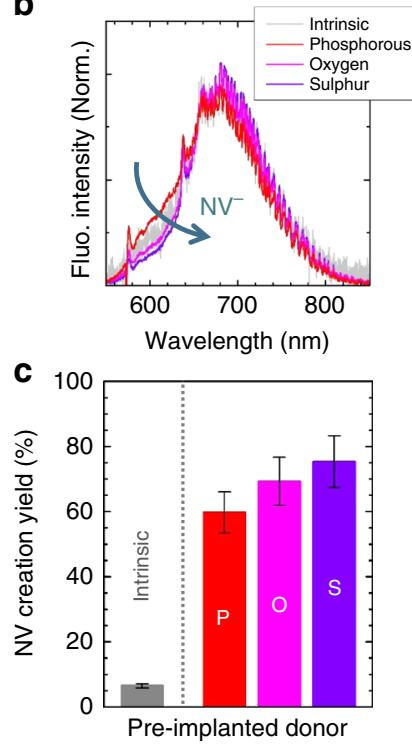

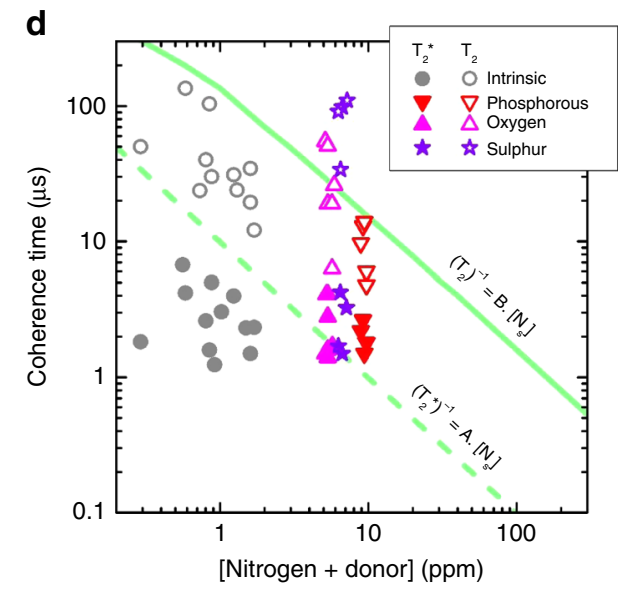

d

Fig. $6 \mathrm{NV}$ centres with different donors. a Confocal fluorescence scans of the NV centres created in the intrinsic and donor-doped areas (nitrogen fluence of $1 \times 10^{10} \mathrm{~cm}^{-2}$, energy of $40 \mathrm{keV}$, annealing at $800^{\circ} \mathrm{C}$ for $4 \mathrm{~h}$ ). The different donors (phosphorous, oxygen, and sulphur) were pre-implanted and annealed at $1200^{\circ} \mathrm{C}$. The laser excitation wavelength is $532 \mathrm{~nm}$ and the detection window $600-700 \mathrm{~nm}$. The fluorescence intensity is plotted in logarithmic scale. The insets are $10 \times 10 \mu \mathrm{m}^{2}$ scans taken at the centre of each implanted spot, plotted in linear scale. b Fluorescence spectra (normalised) from the NV centres in the different areas. The NV- charge state stabilisation increases from $\mathrm{P}$ to $\mathrm{O}$ and $\mathrm{S}$. c NV creation yield vs pre-implanted donor species, together with intrinsic diamond. Donor pre-implantation leads to one order of magnitude increase of NV density and is highest for sulphur. The error bars represent the measurement uncertainties derived from the yield formula given in supplementary Equation 3. $\mathbf{d}$ Coherence times $T_{2}$ and $T_{2}{ }^{\star}$ of several single NV centres as a function of the sum of implanted nitrogen and donor concentration. The $T_{2}{ }^{\star}$ and $T_{2}$ times are obtained, respectively, from the decay of Ramsey (free induction decay) and Hahn echo sequences applied to the single NV centres (with same fit function used as in ${ }^{39}$ ). The lines account

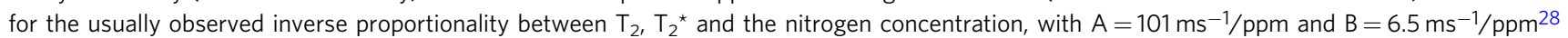

\section{Discussion}

The method and results presented here show that the creation yields of NV and other defect centres are strongly dependent on the doping level of diamond, and that $75 \%$ are achieved using Sulphur doping. The donors modify the centres dynamics of formation during thermal annealing through the charge state and diffusion of the involved species, with a major role likely played by vacancies and hydrogen. The yield is also strongly dependent on the annealing temperature. The highest creations of $\mathrm{NV}, \mathrm{SnV}$, and $\mathrm{MgV}$ centres obtained in donor-doped diamond, for which isolated vacancies were shown to be negatively charged $\mathrm{V}^{-}$, indicates that these are preserved from forming vacancy complexes in profit of $\mathrm{NV}, \mathrm{SnV}$, or $\mathrm{MgV}$. The preliminary carbon implantation also showed that in Boron-doped diamond, where the vacancies are kept neutral, vacancy clustering does take place at lower implantation fluence than in intrinsic or Phosphorousdoped diamond. Charge state tuning of the defects may also be achieved during implantation and/or annealing by applying electrical bias to the sample or using UV illumination.

Highly enhanced creation yields were demonstrated here: $75.3 \%, 8.6 \%$, and $48.4 \%$ for $\mathrm{NV}, \mathrm{SnV}$, and $\mathrm{MgV}$, respectively. Even if not yet fully deterministic, the scalability of $\mathrm{NV}^{-}$based devices is dramatically improved. As an example, to obtain a 5qubit system with the implantation of five nitrogen atoms would require $\sim 1$ million attempts with a $6.5 \%$ yield, whereas only four attempts are needed with a $75 \%$ yield.

Besides, the coherence properties of the NV centres ( $\mathrm{T}_{2}$ times) are improved in Sulphur-doped diamond, which moreover provides the highest $\mathrm{NV}^{-}$stability. The clear correlation between charging of vacancies and improved creation yield of $\mathrm{NV}, \mathrm{SnV}$, and $\mathrm{MgV}$ centres shows that there is plenty of room on the route (s) towards scalable QIP diamond devices and that the deterministic creation of qubits centres may eventually be achieved. The developments of ion implantation techniques are approaching their limits, both in terms of spatial resolution (a few $\mathrm{nm}$ ) and of number of ions, as different methods enable a priori or a posteriori the counting and deterministic implantation of single ions. Furthermore, colour centres can be optically imaged with a resolution below $10 \mathrm{~nm}$ with stimulated emission depletion microscopy. Obviously, diamond inhomogeneities and hydrogen impurities are among the main issues to be overcome in the future. Diamond (area) preselection methods will be developed and traps for hydrogen will for example be designed and implanted to capture the diffusing hydrogen atoms before they may reach and passivate the implanted qubits. Finally, this work gives original insights into $n$-type doping by ion implantation. The recent discovery of luminescent P-related centres together with new opportunities in defect engineering may help reduce the compensation of phosphorous at high concentrations and obtain highly efficient $n$-type diamond, still a major issue for electronic applications.

\section{Methods}

Ion implantation and annealing. Three high purity "quantum grade" (100) CVD single crystal diamonds from Element 6 (with $[\mathrm{B}]<1 \mathrm{ppb}$ and $[\mathrm{N}]<5 \mathrm{ppb}$ ) were used for this work. The strong improve of the NV creation yield in donor-doped diamond was found in all samples (see supplementary Figures 1, 2, 3, and 4 for samples details). The ion implantations were performed using a $100 \mathrm{kV}$ accelerator equipped with a sputter source, an isotopic mass selection, a beam collimation system through movable apertures of different sizes, an (x,y,z) sample stage and an optical microscope. The energies of the different ions were chosen to obtain an average implantation depth of $50 \mathrm{~nm}(28 \mathrm{keV}, 40 \mathrm{keV}$, and $50 \mathrm{keV}$ for $\mathrm{C}, \mathrm{N}$, and $\mathrm{Mg})$ and $25 \mathrm{~nm}(80 \mathrm{keV})$ for $\mathrm{Sn}$. These depths are low enough to ensure a little ion straggling (below $10 \mathrm{~nm}$, as necessary for high-resolution implantation) and large enough to reduce the influence of the diamond surface. For the NV centres, the results are presented for the annealing temperature of $800^{\circ} \mathrm{C}(4 \mathrm{~h})$ in order to compare with previous measurements and literature. The temperature of $800^{\circ} \mathrm{C}$ is generally considered as high enough to enable optimal diffusion of the vacancies to 
reach neighbouring nitrogen atoms and form NV centres efficiently. For the $\mathrm{SnV}$ and $\mathrm{MgV}$ centres, the results are presented for the last annealing step at $120{ }^{\circ} \mathrm{C}$ $(8 \mathrm{~h})$. The full spectral temperature evolution can be found in the supplementary Figure 9.

Donor and acceptor doping. The doping of the sample was achieved prior to the colour centre creation. Areas of different doping types and levels were prepared using ion implantation. Phosphorous (deep donor $E_{\mathrm{D}}=0.57 \mathrm{eV}$ ) and boron (acceptor with $E_{\mathrm{A}}=0.37 \mathrm{eV}$ ) were used in samples 1 and 2. Phosphorous, oxygen and sulphur in sample 3 . The unimplanted diamond area is defined as the intrinsic area. For each dopant, a multiple implantation at different energies and fluences was conducted in order to obtain homogeneous depth concentration profiles of $\sim 2.5 \times 10^{18} \mathrm{~cm}^{-3}$ at the depth of $50 \mathrm{~nm}$, with a thickness of $\sim 50 \mathrm{~nm}$ (see supplementary Figures 2, 3, and 4). The dopant profiles were simulated with SRIM. The samples were then thermally annealed for four hours in vacuum in order to "activate" the dopant atoms as donors or acceptors, respectively (samples 1 and 2 at $1600^{\circ} \mathrm{C}$, sample 3 at $1200^{\circ} \mathrm{C}$ ). The thermal treatment also enables to heal most of the implantation defects. After annealing, the diamonds were exposed to a short oxygen plasma to clean the surface from possible graphite residuals. The samples were then implanted with different atoms for the colour centre screening study.

Optical imaging and spectroscopy and spin measurements. The optical fluorescence imaging and spectroscopic characterisations were conducted on a homemade scanning confocal fluorescence microscope, with an air $(\times 100, \mathrm{NA}=0.95)$ or oil immersion $(\times 60, \mathrm{NA}=1.35)$ objective and two possible laser excitations at $532 \mathrm{~nm}$ and $488 \mathrm{~nm}$. The laser reflection was suppressed by a suitable Notch filter and different spectral filters were used to select the wished fluorescence bands of neutral vacancies $\mathrm{V}^{0}$ (GR1 centre, with ZPL at $\left.741 \mathrm{~nm}\right), \mathrm{NV}$ centres $\left(\mathrm{ZPL}\right.$ of $\mathrm{NV}^{0}$ at $575 \mathrm{~nm}, \mathrm{ZPL}$ of NV ${ }^{-}$at $638 \mathrm{~nm}$ ), SnV centres (ZPL at $620 \mathrm{~nm}$ ) and $\mathrm{MgV}$ centres (ZPL at $557 \mathrm{~nm}$ ). The $\mathrm{T}_{2}$ and $\mathrm{T}_{2}{ }^{*}$ spin coherence time of the single $\mathrm{NV}^{-}$centres were measured on the same setup. Ramsey and Hahn echo sequences were conducted, with microwave pulses applied using a thin wire located close to the implanted centres.

Determination of the creation yield. The creation yield of the different centres was determined in two different ways, depending on their density. If the centres could be resolved (typically for densities below $3-4$ centres per $\mu \mathrm{m}^{2}$ ) they were simply counted within a reference area, and compared with the density (fluence) of implanted ions. For higher colour centres densities, single centres were taken as reference and scaled to the dense implanted areas (see supplementary information), taking background fluorescence into account.

\section{Data availability}

The data that support the findings of this study are available from the corresponding author upon reasonable request.

Received: 24 April 2019; Accepted: 11 September 2019;

Published online: 31 October 2019

\section{References}

1. Ladd, T. D. et al. Quantum computers. Nature 464, 45-53 (2010).

2. Rondin, L. et al. Magnetometry with nitrogen-vacancy defects in diamond. Rep. Prog. Phys. 77, 056503 (2014).

3. Dolde, F. et al. Electric-field sensing using single diamond spins. Nat. Phys. 7, 459-463 (2011).

4. Kucsko, G. et al. Nanometre-scale thermometry in a living cell. Nature 500, 54-58 (2013)

5. Staudacher, T. et al. Nuclear magnetic resonance spectroscopy on a (5nanometer $)^{3}$ sample volume. Science 339, 561-563 (2013).

6. Jacques, V. et al. Experimental realization of Wheeler's delayed-choice gedanken experiment. Science 315, 966-968 (2007).

7. Hensen, B. et al. Loophole-free Bell inequality violation using electron spins separated by 1.3 kilometres. Nature 526, 682-686 (2015).

8. Dolde, F. et al. Room-temperature entanglement between single defect spins in diamond. Nat. Phys. 9, 139-143 (2013).

9. Lühmann, T. et al. Screening and engineering of colour centres in diamond. $J$. Phys. D: Appl. Phys. 51, 483002 (2018).

10. Pezzagna, S. et al. Nanoscale engineering and optical addressing of single spins in diamond. Small 6, 2117-2121 (2010).

11. Riedrich-Möller, J. et al. Nanoimplantation and Purcell enhancement of single nitrogen-vacancy centers in photonic crystal cavities in diamond. Appl. Phys. Lett. 106, 221103 (2015).

12. Schnitzler, W. et al. Deterministic ultracold ion source targeting the Heisenberg limit. Phys. Rev. Lett. 102, 070501 (2007).
13. Räcke, P., Spemann, D., Gerlach, J. W., Rauschenbach, B. \& Meijer, J. Detection of small bunches of ions using image charges. Sci. Rep. 8, 9781 (2018).

14. Groot-Berning K. et al. Deterministic single ion implantation of rare-earth ions for nanometer-resolution color-center generation. Phys. Rev. Lett. 123, 106802 (2019).

15. Jamieson, D. N. et al. Controlled shallow single-ion implantation in silicon using an active substrate for sub-20-keV ions. Appl. Phys. Lett. 86, 202101 (2005).

16. Davies, G. \& Hamer, M. F. Optical studies of the $1.945 \mathrm{eV}$ vibronic band in diamond. Proc. R. Soc. A 348, 285-298 (1976).

17. Zaitsev A. M. Optical properties of diamond, (Berlin Springer, 2001).

18. Davies, G., Lawson, S. C., Collins, A. T., Mainwood, A. \& Sharp, S. J. Vacancy related centers in diamond. Phys. Rev. B 46, 13157-13170 (1992).

19. Collins, A. T. \& Kiflawi, I. The annealing of radiation damage in type Ia diamond. J. Phys. Condens. Matter 21, 364209 (2009).

20. Pezzagna, S., Naydenov, B., Jelezko, F., Wrachtrup, J. \& Meijer, J. Creation efficiency of nitrogen-vacancy centres in diamond. New J. Phys. 12, 065017 (2010).

21. Yamamoto, T. et al. Extending spin coherence times of diamond qubits by high-temperature annealing. Phys. Rev. B 88, 075206 (2013).

22. Yamamoto, T. et al. Strongly coupled diamond spin qubits by molecular nitrogen implantation,. Phys. Rev. B 88, 201201(R) (2013).

23. Acosta, V. M. et al. Diamonds with a high density of nitrogen-vacancy centers for magnetometry applications. Phys. Rev. B 80, 115202 (2009).

24. Becker, S., Raatz, N., Jankuhn, S., John, R. \& Meijer, J. Nitrogen implantation with a scanning electron microscope. Sci. Rep. 8, 32 (2018).

25. Chen, Y.-C. et al. Laser writing of individual atomic defects in a crystal with near-unity yield. Optica 6, 662-667 (2019).

26. Mainwood, A. Nitrogen and nitrogen-vacancy complexes and their formation in diamond. Phys. Rev. B 49, 7934-7940 (1994).

27. Deák, P., Aradi, B., Kaviani, M., Frauenheim, T. \& Gali, A. Formation of NV centers in diamond: a theoretical study based on calculated transitions and migration of nitrogen and vacancy related defects. Phys. Rev. B 89, 075203 (2014)

28. Barry J. F. et al. Sensitivity optimization for NV-diamond magnetometry. Preprint at https://arxiv.org/abs/1903.08176 (2019).

29. Groot-Berning, K. et al. Passive charge state control of nitrogen-vacancy centres in diamond using phosphorous and boron doping. Phys. Stat. Sol. A 211, 2268-2273 (2014).

30. Doi, Y. et al. Pure negatively charged state of the NV center in n-type diamond. Phys. Rev. B 93, 081203(R) (2016).

31. Manson, N. B. et al. $\mathrm{NV}^{-}-\mathrm{N}^{+}$pair centre in $1 \mathrm{~b}$ diamond. New J. Phys. 20, 113037 (2018)

32. Mittiga, T. et al. Imaging the local charge environment of nitrogen-vacancy centers in diamond. Phys. Rev. Lett. 121, 246402 (2018).

33. Edmonds, A. M. et al. Production of oriented nitrogen-vacancy color centers in synthetic diamond. Phys. Rev. B 86, 035201 (2012).

34. Stacey, A. et al. Depletion of nitrogen-vacancy color centers in diamond via hydrogen passivation. Appl. Phys. Lett. 100, 071902 (2012).

35. Lesik, M. et al. Production of bulk NV centre arrays by shallow implantation and diamond CVD overgrowth. Phys. Stat. Sol. A 213, 2594-2600 (2016).

36. Saguy, C. et al. Diffusion of hydrogen in undoped, p-type and n-type doped diamonds. Diam. Relat. Mater. 12, 623-631 (2003).

37. Chevallier, J. et al. Hydrogen in n-type diamond. Diam. Relat. Mater. 11, 1566-1571 (2002).

38. Arnault, J. C. et al. Enhanced deuterium diffusion in boron doped monocrystalline diamond films using bias-assisted MPCVD. Phys. Lett. A 374, 3254-3257 (2010)

39. Fávaro de Oliveira, F. et al. Tailoring spin defects in diamond by lattice charging. Nat. Commun. 8, 15409 (2016).

40. Stenger, I. et al. Quantitative analysis of electronic absorption of phosphorous donors in diamond. Diam. Relat. Mater. 74, 24-30 (2017).

41. Czelej, K., Zemla, M. R., Kaminska, P., Spiewak, P. \& Kurzydlowski, K. J. Clustering of hydrogen, phosphorous, and vacancies in diamond: a density functional theory analysis. Phys. Rev. B 98, 075208 (2018).

42. Prins, J. F. $n$-type semiconducting diamond by means of oxygen-ion implantation. Phys. Rev. B 61, 7191-7194 (2000).

43. Wang, L. G. \& Zunger, A. Phosphorous and sulphur doping of diamond. Phys. Rev. B 66, 161202(R) (2002).

44. Collins, A. The Fermi level in diamond. J. Phys. Cond. Mater. 14, 3743-3750 (2002).

45. Ziegler J. The stopping and range of ions in matter, SRIM-2008, online at http://srim.org (2008).

46. Miyazaki, T. \& Yamasaki, S. Ab initio energetics of phosphorous related complex defects in synthetic diamond. Phys. B 376, 304-306 (2006).

47. Goss, J. P., Briddon, P. R., Rayson, M. J., Sque, S. J. \& Jones, R. Vacancy impurity complexes and limitations for implantation doping of diamond. Phys. Rev. B 72, 035214 (2005). 
48. Hofsäss, H., Dalmer, M., Restle, M. \& Ronning, C. Substitutional phosphorus doping of diamond by ion implantation. J. Appl. Phys. 81, 2566-2569 (1997).

49. Goss, J. P. et al. Theory of hydrogen in diamond. Phys. Rev. B 65, 115207 (2002).

50. Rose, B. C. et al. Observation of an environmentally insensitive solid-state spin defect in diamond. Science 361, 60-63 (2018).

51. Ditalia Tchernij, S. et al. Single-photon-emitting optical centers in diamond fabricated upon Sn implantation. ACS Photonics 4, 2580-2586 (2017).

52. Iwasaki, T. et al. Tin-vacancy quantum emitters in diamond. Phys. Rev. Lett. 119, 253601 (2017).

53. Thiering, G. \& Gali, A. Ab initio magneto-optical spectrum of group-IV vacancy color centers in diamond. Phys. Rev. X 8, 021063 (2018).

54. Alkahtani, M. et al. Tin-vacancy in diamonds for luminescent thermometry. Appl. Phys. Lett. 112, 241902 (2018).

55. Schröder, T. et al. Scalable focused ion beam creation of nearly lifetime-limited single quantum emitters in diamond nanostructures. Nat. Comm. 8, 15376 (2016).

56. Zhou, Y. et al. Direct writing of single germanium vacancy center arrays in diamond. New J. Phys. 20, 125004 (2018).

57. Broadway, D. A. et al. Spatial mapping of band bending in semiconductor devices using in situ quantum sensors. Nat. Electron. 1, 502-507 (2018).

\section{Acknowledgements}

We acknowledge the support from the European Union (ASTERIQS, MICROSENS) as well as the Deutsche Forschungsgemeinschaft and the Volkswagen Stiftung.

\section{Author contributions}

T.L. performed the implantations and spectroscopy. R.J. performed the measurements of coherence times. R.W. had the idea to use Sulphur. J.M. and S.P. designed the concept idea and supervised the work. S.P. wrote the paper. All authors revised the manuscript.

\section{Competing interests}

The authors declare no competing interests.

\section{Additional information}

Supplementary information is available for this paper at https://doi.org/10.1038/s41467019-12556-0.

Correspondence and requests for materials should be addressed to S.P.

Peer review information Nature Communications thanks the anonymous reviewers for their contribution to the peer review of this work.

Reprints and permission information is available at http://www.nature.com/reprints

Publisher's note Springer Nature remains neutral with regard to jurisdictional claims in published maps and institutional affiliations.

(c) (i) Open Access This article is licensed under a Creative Commons Attribution 4.0 International License, which permits use, sharing, adaptation, distribution and reproduction in any medium or format, as long as you give appropriate credit to the original author(s) and the source, provide a link to the Creative Commons license, and indicate if changes were made. The images or other third party material in this article are included in the article's Creative Commons license, unless indicated otherwise in a credit line to the material. If material is not included in the article's Creative Commons license and your intended use is not permitted by statutory regulation or exceeds the permitted use, you will need to obtain permission directly from the copyright holder. To view a copy of this license, visit http://creativecommons.org/ licenses/by/4.0/.

(c) The Author(s) 2019 